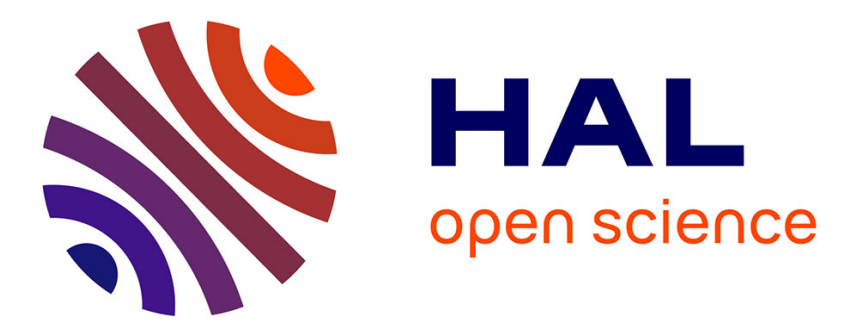

\title{
Isabelle Stengers, William James, Une autre science est possible! Manifeste pour un ralentissement des sciences (suivi de: Le poulpe du doctorat) \\ Odile Camus
}

\section{- To cite this version:}

Odile Camus. Isabelle Stengers, William James, Une autre science est possible! Manifeste pour un ralentissement des sciences (suivi de: Le poulpe du doctorat). 2018. hal-02530441

HAL Id: hal-02530441

https://hal-normandie-univ.archives-ouvertes.fr/hal-02530441

Submitted on 2 Apr 2020

HAL is a multi-disciplinary open access archive for the deposit and dissemination of scientific research documents, whether they are published or not. The documents may come from teaching and research institutions in France or abroad, or from public or private research centers.
L'archive ouverte pluridisciplinaire HAL, est destinée au dépôt et à la diffusion de documents scientifiques de niveau recherche, publiés ou non, émanant des établissements d'enseignement et de recherche français ou étrangers, des laboratoires publics ou privés. 
Isabelle Stengers, William James,

\section{Une autre science est possible ! \\ Manifeste pour un ralentissement des sciences \\ (suivi de Le poulpe du doctorat)}

Odile Camus

1 La première partie de ce manifeste réunit cinq textes d'Isabelle Stengers, textes issus pour la plupart d'exposés produits entre 2009 et 2012. Tous mettent en exergue l'urgence de concevoir la science et de pratiquer la recherche scientifique suivant une perspective radicalement alternative dont le mot d'ordre de « ralentissement » annonce la teneur. L’urgence, c’est de rompre avec un progrès devenu « développement insoutenable » (p. 12), de s’extraire de la logique capitaliste qui nous entraîne vers un désastre social et écologique. L’auteure rend compte de cette logique telle qu'elle opère dans l'université, institution où se fabriquent la science et les chercheurs, et qui est ici vue du dedans; et c'est d'abord aux universitaires qu'elle s'adresse (voir p.121). Chacun de ces textes se focalise plus particulièrement sur une idée, mais les principaux arguments traversent l'ensemble du recueil.

2 Concevoir autrement la science, c’est en premier lieu reconsidérer l’opposition entre rationalité scientifique et opinion, comme entre faits et valeurs. Cette opposition, généralement tenue pour évidente et ce faisant non questionnée, assoit l’autorité d’un savoir excluant un public perçu comme incapable de se l'approprier. Contre ces aprioris, l'auteure en appelle à une « intelligence publique » des sciences 1 . Selon elle, les scientifiques eux-mêmes ont besoin que se développe ce « rapport intelligent » aux sciences, par exemple pour se défendre contre les « marchands de doute » opérant sur Internet. 
3 Le deuxième texte interroge l' « étoffe du chercheur », d'abord en tant que construction genrée. Par exemple : « D’une femme que ses responsabilités familiales handicapent, on dira souvent que le fait même qu'elle ait choisi d'assumer de telles responsabilités prouve qu’elle n’avait peut-être pas l'“étoffe” du véritable chercheur » (p. 29). Cette étoffe, définie par la négative (Cf. le non-marquage du genre supérieur), produit un ethos « qui n’a pas de rapport direct avec la capacité de faire de la recherche » (p. 30), mais « qui se présente comme synonyme d'esprit scientifique, et qui aboutit aujourd'hui à une définition de l’excellence “basée sur les faits” » (p. 37), lesquels fonderaient l'« objectivité ». Or le « verdict des faits » et l' « autorité de la preuve », censés rendre décidable toute question, seraient une imposture assise notamment sur l'exclusion des questions qui ne peuvent se définir en des termes permettant « à des données objectivement mesurables d'évaluer et de trancher » (p. 36). L' « étoffe du chercheur », ce somnambule qu'il ne faut surtout pas réveiller, suppose en effet sa « chasteté » vis-à-vis des « grandes questions » disqualifiées comme non scientifiques - questions telles que celles portant sur la civilisation dans laquelle nous nous trouvons.

4 Le troisième texte se focalise sur l'évaluation de la recherche, régie par une «pseudo-loi du marché », laquelle s’installe là où le marché ne peut être défini en termes de transactions économiques, et qui fait exister la fiction de l'offre et de la demande. Compétition pour l' « excellence », mise en conformité avec ce qu'imposent les revues de rang $\mathrm{A}$, soumission à des critères contradictoires avec ce qui donne sens à l'activité des chercheurs, évaluation par les « collègues compétents » (les pairs) qui ne feront en somme qu’une vérification de spécialistes... Ces modalités relèvent d’un système d'évaluation rapide, inventé « par et pour les sciences rapides » (p. 54) - c'est-à-dire, à l'origine, les sciences expérimentales -, et inscrit dans un modèle de connaissance cumulative à propos d'un monde considéré comme donné. Pour se libérer de ce modèle, l'auteure en appelle à la pluralité des sciences et des modes d'évaluation. Elle fait notamment référence aux sciences sociales, dans lesquelles l'objet doit être pensé 
autrement que comme « matière à preuve ».

5 Le « plaidoyer pour une science slow » que constitue le quatrième texte peut être lu comme un appel à la résistance dans les universités - où prévaut la méfiance envers « ce qui pourrait ressembler à une "politisation” de la science » (p. 86). L’auteure invite à penser « le possible contre le probable » (p. 92), dans une démarche de « consultation », que nous avons désapprise. Refuser les « outils néomanagériaux [...] voués à redéfinir ce qui compte comme connaissance » (p. 84), ainsi que les « questions toutes faites » (p. 93), s’intéresser à des questions qui importent dans d'autres disciplines, « penser ensemble » et « faire hésiter chacun », c’est en somme un rapport démocratique au savoir qu'il nous faut prendre le temps de construire.

6 Avec le dernier texte, le ralentissement apparaît clairement comme la seule option pour rendre possible un avenir viable - paradoxalement, car il y a urgence : Gaïa, « celle-quifait-intrusion » 3 , met en question l'avenir de tous les habitants de la Terre, et nous impose des questions que nos pratiques et nos institutions ne nous permettent pas de formuler. L’incompatibilité de l'écologie politique avec la logique capitaliste exige la rupture d'avec cette dernière, rupture qui doit prendre la forme d’une « civilisation » des pratiques, et en premier lieu des pratiques scientifiques. Nous poser à nous, chercheurs, la question de notre civilisation, nous réapproprier nos pratiques : réinventer la valeur de nos productions et nous soucier de leurs conséquences, nous interroger sur ce que nous faisons de nos savoirs, et ne plus nous protéger des questions du public concerné constituent quelques conditions du nécessaire « ralentissement cosmopolitique ».

7 La deuxième partie de l’ouvrage est consacrée à un article de 1903 de William James, traduit et présenté par Thierry Drumm. Le thème en est l’institutionnalisation du doctorat, thème à même d’illustrer le propos précédent. D’ailleurs la psychologie pragmatique de James, souvent mal comprise et dont un aperçu éclairant est donné ici, se 
révèle elle-même comme un outil particulièrement adapté pour penser le ralentissement des sciences. Car le pragmatisme n'est pas une philosophie de l'action, mais bien plutôt une philosophie de l’hésitation, de la pensée (toujours située, et indissociable de l’expérience) en tant que celle-ci permet de suspendre la réaction immédiate.

8 Centrée sur les conséquences des situations plutôt que sur les causes, la lecture pragmatique de l'institution doctorale permet à James d’apprécier la valeur de cette institution à partir de l'expérience de ses effets. L'image du poulpe (titre de son article) réfère à sa conception réticulaire du réel (et de la pensée) : rien n’agit isolément. Le « jeu professoral » peut ainsi être décrit comme un réseau dans lequel les connexions deviennent exclusives au point d'interdire de nouvelles connexions. Peu à peu, l’âme possédée par le poulpe « n’est plus capable d’inventer ce qu’elle aura été, elle ne tend plus qu'à se conformer au modèle qu'on lui propose » (p. 170). Par ailleurs James, partisan du « nivellement », s’oppose à tout modèle hiérarchique et à toute distinction honorifique. Or les études, organisées selon une progression visant un but, amènent à définir pour seul enjeu de la connaissance une carrière à faire. En même temps, cette organisation contrarie le cheminement de la pensée - qui n’est pas linéaire, mais fabrique un réseau. Ainsi « le doctorat, en tant qu'il sanctionne l'acquisition d'un niveau d'études, contrevient [...] nécessairement à l’apparition de pensées originales » (p. 180). Le poulpe favorise un « lissage du savoir » et la production d'une « uniformité bien pensante » (p. 198).

9 L'article de James lui-même relate la découverte, par les directeurs d'une institution universitaire, qu’un chargé de cours en littérature ne possède pas ce « bibelot académique » (p. 199) qu'est le doctorat. La renommée de l'institution risquerait d'en pâtir, le doctorat étant considéré comme un « instrument publicitaire ». Pour conserver son emploi, le chargé de cours soutient donc une thèse - correspondant à sa formation initiale, en l'occurrence la philosophie, et non à la matière de son enseignement, mais nul n’y trouve à redire. À partir de cette expérience, James relève les maux dérivés de ce 
système, par exemple l’absence de rapport entre le titre de docteur et les qualités d’enseignant. Et cet « esprit de snobisme académique » dont témoigne le récit a ses victimes, abusées par la croyance populaire en la valeur de nos diplômes. De manière générale, les examens d’État, notamment de par leur « prolifération tyrannique » (p. 208), constituent un frein à la spontanéité personnelle. Pour limiter la mainmise du poulpe du doctorat, James propose (entre autres) de décerner automatiquement le doctorat « pour une certaine quantité de temps consacré à un travail patient dans un certain domaine de savoir » (p. 209).

10 Voilà donc une lecture revigorante, tant de par la mise à plat, sans détour, de « vérités qui dérangent », que par le potentiel de résistance de ce mot d’ordre : prendre le temps, ralentir.

\section{NOTES}

1. Elle évoque par exemple le rôle des groupes contestataires dans l'instauration d'un rapport plus lucide aux OGM (p. 16).

2. En ethnologie, par exemple, le chercheur apprend quelque chose de son objet plutôt que sur lui. Notons que le terme d'objet n'est utilisé dans ce compte rendu que par commodité ; il ne se trouve pas sous la plume d'Isabelle Stengers.

3. Et dont la dénomination est choisie pour susciter un « nous », contre le « on » qui « sait » (p. 115). 